\title{
Exploring the Perception of Information Communication Tools Application in Agricultural Education
}

\author{
Suman Saha $^{1 *}$, S. D. Mukhopadhyay ${ }^{2}$, K. Pradhan $^{3}$ and S. K. Acharya ${ }^{4}$
}

${ }^{1} S R F$, ICAR-NASF, Uttar Banga Krishi Viswavidyalaya, Pundibari, Coochbehar, WB, India

${ }^{2}$ Department of Agricultural Extension, PSB, Visva Bharati, Sriniketan, WB, India

${ }^{3}$ Department of Agricultural Extension, Uttar Banga Krishi Viswavidyalaya, Pundibari, Coochbehar, WB, India

${ }^{4}$ Department of Agricultural Extension, Bidhan Chandra Krishi Viswavidyalaya, Mohanpur, Nadia, WB, India

*Corresponding author

\section{A B S T R A C T}

\section{Keywords \\ Community differential, Computer tools, Gap Index, ICT tools, Perception \\ Article Info \\ Accepted: \\ 18 December 2020 \\ Available Online: \\ 10 January 2021}

\begin{abstract}
In evolving and modernizing higher education in agriculture, the role of ICT is integral and essential. In education, the process of digitization is gaining new dimension and scales to make it active, effective and applicable. In higher educational sphere the role of computer as well as ICT gadgets are playing the pivotal role. The present study has focused on the application of computer vis-a-vis ICT tools by the students, research scholars and faculties at Palli Siksha Bhavana. A score of 270 respondents comprising of students, scholars and faculties of Palli Siksha Bhavana have been interrogated to elicit the status and application of different ICT tools by the students, research scholars and faculties. The study reveals that different community categories have got differential level of ICT tools. There has been and upscaling of computer application when the respondents shifted from Secondary to Under Graduate level. Albeit there has been a higher intensity of computer application among the respondents, the Gap Index has also be widening in terms of perception and psychological constraint in the realm of computer application at proper level and direction.
\end{abstract}

\section{Introduction}

Computers are the part and parcel of life in today's world. From the schedule starting from early bed to late night, all the activities are online. Today whole world is shrinking to technology and nobody is far away in any aspect. One can talk of any enterprise, everything is linked to technology. India, being an agrarian country where livelihood of
$65 \%$ population depends on agriculture is the sector where computers and its applications are adopted in a less majority as compare to other developed countries. Our agriculture is mechanized but our farmers are lacking knowledge regarding role of computer applications in agriculture. It has been rightly said that computers and their applications changed the structure of most traditional occupations like agriculture. Technological 
advances have brought about drastic changes in agriculture, its subsidiary occupations like; fish farming, dairy farming, bee keeping, mushroom farming which results in a tremendous increase in productivity. Computer operated software for different crops are available to assess yield performance by using different parameters. Computers are used for record-keeping related to cost of production, transport, agricultural operations, and in the estimation and calculation of profit and or loss. The uses of computers in agriculture do have some real constraints such as, the lack of hardware and software infrastructure, training and skills, and research priorities (Grewal et al., 2015). The Internet namely various portals in agricultural science, most government websites provide this kind of information free of cost, covering agricultural land masses helps in interaction among the farmers and development officials working in rural sector both in agriculture and in other rural developmental programme. The use of computers and application of ICT had become common phenomena in every walk of life. Corporate houses, Banks, Private business establishments, Government to Citizen have been benefited by use of this new technology (Das et al., 2011). This results in an exchange of knowledge and serves as guidance for farmers or rural people to improve production as well as their livelihood. Mechanization has reduced human or animal effort and increased the speed and quality of production. By linking communication technologies to market exchanges in commercial centers, even small farmers can overcome the enormous informational asymmetries that limit their bargaining power in traditional supply chains. The revolution in mobile phones is helping the small farmers to get information about crop prices and input prices and other related information on agriculture. The use of computer hardware, such as laptops, scanners, printers, modems and internet, has exploded in recent decades. Software designed to increase management efficiency abound. The use of computer applications within teaching and learning of Management could have positive effects, on one hand, and negative effects, on the other hand. Though using computer applications in education has shown educational benefits, changing traditional teaching and learning is yet a challenging process (Andreea, 2008). By making efficient use of computer applications by the development officials specially working in rural sector can increase the productivity as well as livelihood of the rural peoples. In India, agriculture is still remaining as a public sector endeavor where, government, both national and states are the major instrument to bring about positive changes in the production and productivity of crops and lands. Accordingly government has steady developmental network covering the entire country right from village level to the national level to reach with the farmers different developmental inputs like, technology, different developmental projects, management of overall agriculture etc. Computing has an important role in the outof-school activities of many children and that it is often a social activity. The study had suggests the need for introducing the educational programs for agricultural extension staff, their supervisors and managers to make them realized the importance of the role of computers in extension work (Ioanna, 2009). The network is maintained by agricultural scientists, extension and development professionals. For that matter, primarily it is essential that the agricultural students and academicians, scientists, extension and development professionals working in field of agricultural and rural development should have sufficient knowledge and efficiency in use of computer. Which not only help reaching all the farmers and farming situations in minimum time with minimum expenditure but also provides an 
opportunity to develop and use decision support system in different aspects of agriculture which can in turn help the farmer to take appropriate decision for their farming situation. Keeping the background in mind the present study has envisaged with an aim to analyse the extent of involvement in different computer uses by agricultural students and academic professionals as well as to identify the constraints faced by the agricultural students and academic professionals while using computer.

\section{Materials and Methods}

The present study has been conducted among the students (UG, PG and Ph.D) and faculty members of the Institute of Agriculture, Visva-Bharati, Sriniketan and KVK Scientists of Rathinda KVK, Visva Bharati, Sriniketan. The purposive and random sampling procedures were followed to draw the sample from the population. The sample size for the study is 270 (UG students-132, PG students 61, PhD students-30, Faculty members-40, KVK scientists-7). The dependent and independent variables for the study were operationalised and measured with the help of slightly modified pre constructed scales. The data were collected with the help of pretested structured interview schedule constructed for the study through personal interview method. The data were processed into percentage, mean and gap indexing for drawing a definite conclusion from the study.

\section{Results and Discussion}

The following results were enumerated through the collected data in the present study:

\section{Age of the respondents}

Table-1 represents the age of the respondents in ten class intervals, i.e., Up to 20 years, 21-
25 years, 25-30 years, 31-35 years, 36-40 years, 41-45years, 46-50 years, 51-55 years, 56-60 years and 61-65 years respectively. From the table it can be observed that majority of respondents fall under the age group 21-25 years $(43.33 \%)$ followed by the age group up to 20 years (29.25\%), age group 41-45 (15.18\%), age group 25-30 (8.52\%), age group 36-40 (4.85\%), age group 31-35 $(4.81 \%)$, age group $51-55(2.22 \%)$, age group $56-60(1.48 \%)$ and the rest two class interval i.e., age group 46-50 and 61-65 (1.85\%) were having the same numbers. From the above context, it is found that most of the respondents are of younger age.

\section{Caste orientation of the respondents}

As per the caste orientation of the respondents, it can be observed from the table-2 that in UG most of the respondents belong to General Categories (24.07\%) followed by OBC (15.56\%), SC (4.81\%), ST $(2.59 \%)$ and Minority (1.85\%). In case of PG majority of the respondents belong to $\mathrm{OBC}$ (9.25\%) followed by General (6.67\%), SC (3.33\%), ST (2.22\%) and Minority (1.11\%). While in Ph.D both General and OBC $(4.07 \%)$ were the majority followed by Minority and ST $(0.74 \%)$ and rest $(1.11 \%)$ respondents belong to SC. Among faculty members it is found that majority of respondents belong to General category $(12.22 \%)$ followed by SC (3.33\%), OBC $(1.11 \%)$, ST $(0.74 \%)$ and Minority $(0.37 \%)$ respectively. When taking the total respondents together it was found that General Category (47.03\%) was majority followed by OBC (30\%), SC (12.66\%), ST $(6.24 \%)$ and Minority $4.07 \%)$ in descending order.

\section{Gender distribution of the respondents}

It can be observed from the table- 3 that out of 270 respondent 181 were male from which 78 
(28.89\%) were from UG, $41(15.18 \%)$ from PG, 19 (7.03\%) from Ph.D scholar and 43 $(15.92 \%)$ were faculty member. It is also observed that among the respondents 89 were female of which $54(20 \%)$ were in UG, 20 (7.40\%) in PG, 10 (3.70\%) Ph.D scholar and 5 (1.85) faculty members respectively.

\section{Religion of respondents}

Among 270 respondents, a majority reported their religion as Hindu (124 respondent, $45.92 \%$ ) among UG followed by 55 respondents $(20.37 \%)$ among PG, 26 respondents (9.62\%) among Ph.D and 46 respondents (17.03\%) among Faculty members. Totally it is observed that among 270 respondents, a majority 253 respondents (93.73\%) reported their religion as Hindu, followed by Muslim 15 (5.56\%) respondents and Christianity 3 respondents (1.11\%).

\section{Educational level of the respondents}

Education is one of the most important characteristics that might affect the person's attitudes and the way of looking and understanding any particular social phenomena. In this way, the response of an individual is likely to be determined by his educational status and therefore it becomes imperative to know the educational background of the respondents. From the above table it is observed that, a majority of respondents $132(48.88 \%)$ is continuing and educated up to UG followed by 61 respondents $(22.59 \%)$ in PG and 30 respondents $(11.11 \%)$ in $\mathrm{Ph} . \mathrm{D}$ respectively. It is also revealed from the above table that all the faculty members 47 in numbers $(17.40 \%$ of the total sample) were having Ph.D degree.

\section{Family educational status}

Family education is an important aspect for their children in proper guiding, motivating and directing for higher education. Higher level of parental education helps their children to create conducive situation which will have good understanding regarding various modern ways of advancement of their children. They motivate their children to make best use of all the means of development. To ascertain the family educational level a scale, as described in the methodology chapter has been used.

From the above table- 6 it can be observed that 98 respondents $(36.29 \%)$ were having family educational score more than 6 i.e. up to college and above, followed by 74 respondents $(27.40 \%)$ up to high school, 71 respondents (26.29\%) up to middle school, 24 respondents (8.89\%) up to primary school and only one respondent $(0.37 \%)$ was found to have family educational status up to can read and write only.

\section{Family type of the respondents}

The type of family in which person lives and gets socialized has immense importance in deciding his values, beliefs and behavioral patterns which are likely to affects his or her attitudes towards a particular problem, hence the family type plays its own role in giving the response of an individual and therefore it was thought to be important to understand the family type of the respondents. Distribution of the type of family of the respondents were analysed and presented in table below.

From table-6.1.6 it can be observed that majority of the respondents $143(53.66 \%)$ were living in nuclear family and remaining 125 respondents $(46.34 \%)$ belonged to the joint family.

\section{Family size}

So far number of family members are concerned it is found from the table 8 that 
majority of the respondents $(33.7 \%)$ were having family members more than 7 followed by up to 4 numbers of members $(20.74 \%)$, $15.92 \%$ were having up to 5 members, $12.96 \%$ and $12.59 \%$ were having up to 6 and 3 members in their family.

\section{Possession of computer related material by the respondents}

Table 9 represents the possession of computer related material by the respondents. It is found from the table that out of the total respondents $40.74 \%$ had own desktop, $55.56 \%$ had laptop, $54.81 \%$ had modem, $100 \%$ had mobile phone and $74.81 \%$ possessed smart phone. Besides, it was also observed that $17.4 \%$ of the total respondents had official desktop, $18.51 \%$ had official laptop, $17.4 \%$ had official modem.

As revealed from the table it can observed that majority of the respondents (44.44\%) have average annual income within the range of Rs.2.5 to Rs 5 lakh, followed by (18.51\%) of the respondents annual income ranges up to Rs.2.5 lakh, (13.70\%) between Rs 5 to 7.5 lakh, (7.40\%) between Rs.7.5 to 10 lakh, (7.03\%) between Rs. 10 to 12.5 lakh and $(8.89 \%)$ were having annual income more than Rs. 12.5 .

Table.1 Distribution of the respondents according to their age $n=270$

\begin{tabular}{|c|c|}
\hline Age & No.(\%) \\
\hline Up to 20 & $79(29.25 \%)$ \\
\hline $\mathbf{2 1 - 2 5}$ & $117(43.33 \%)$ \\
\hline $\mathbf{2 5 - 3 0}$ & $23(8.52 \%)$ \\
\hline $\mathbf{3 1 - 3 5}$ & $4(4.81 \%)$ \\
\hline $\mathbf{3 6 - 4 0}$ & $13(4.85 \%)$ \\
\hline $\mathbf{4 1 - 4 5}$ & $14(15.18 \%)$ \\
\hline $\mathbf{4 6 - 5 0}$ & $5(1.85 \%)$ \\
\hline $\mathbf{5 1 - 5 5}$ & $6(2.22 \%)$ \\
\hline $\mathbf{5 6 - 6 0}$ & $4(1.48 \%)$ \\
\hline $\mathbf{6 1 - 6 5}$ & $5(1.85 \%)$ \\
\hline
\end{tabular}

Table.2 Distribution of the respondents according to their caste $n=270$

\begin{tabular}{|c|c|c|c|c|c|}
\hline Categories & UG(132) & PG(61) & PhD(30) & Faculty(47) & Total \\
\hline GEN & $65(24.07 \%)$ & $18(6.67 \%)$ & $11(4.07 \%)$ & $32(11.85 \%)$ & $126(46.67 \%)$ \\
\hline OBC & $42(15.56 \%)$ & $24(8.89 \%)$ & $11(4.07 \%)$ & $3(1.11 \%)$ & $80(29.62 \%)$ \\
\hline SC & $13(4.81 \%)$ & $9(3.33 \%)$ & $4(1.48 \%)$ & $9(3.33 \%)$ & $35(12.66 \%)$ \\
\hline ST & $7(2.59 \%)$ & $6(2.22 \%)$ & $2(0.74 \%)$ & $2(0.74 \%)$ & $17(6.24 \%)$ \\
\hline Minority & $5(1.85 \%)$ & $3(1.11)$ & $2(0.74 \%)$ & $1(0.37 \%)$ & $11(4.07 \%)$ \\
\hline
\end{tabular}

Table.3 Gender Distribution of the Respondents $n=270$

\begin{tabular}{|c|c|c|c|c|}
\hline Gender & UG & PG & PhD & Faculty \\
\hline Male (181) & $78(28.89 \%)$ & $41(15.18 \%)$ & $20(7.40 \%)$ & $43(15.92 \%)$ \\
\hline Female (89) & $54(20 \%)$ & $20(7.40 \%)$ & $10(3.70 \%)$ & $5(1.85 \%)$ \\
\hline
\end{tabular}


Table.4 Distribution of the respondent according to religion $n=270$

\begin{tabular}{|c|c|c|c|c|c|}
\hline Religion & UG & PG & PhD & Faculty & Total \\
\hline Hindu & $124(45.92 \%)$ & $55(20.37 \%)$ & $28(10.37 \%)$ & $45(16.67 \%)$ & $252(93.33 \%)$ \\
\hline Muslim & $8(2.96 \%)$ & $5(1.85 \%)$ & $1(0.37 \%)$ & $1(0.37 \%)$ & $15(5.56 \%)$ \\
\hline Christianity & 0 & $1(0.37 \%)$ & $1(0.37 \%)$ & $1(0.37 \%)$ & $3(1.11 \%)$ \\
\hline Total & 132 & 61 & 30 & 47 & 270 \\
\hline
\end{tabular}

Table.5 Distribution of the respondent according to their level of education $n=270$

\begin{tabular}{|c|c|c|}
\hline Degree & Continuing & Awarded \\
\hline UG (HS Passed) & $132(48.88 \%)$ & \\
\hline PG (Graduate) & $61(22.59 \%)$ & \\
\hline PhD (PG) & $30(11.11 \%)$ & \\
\hline Faculty & & $47(17.40 \%)$ \\
\hline
\end{tabular}

Table.6 Distribution of respondent according to their family education $n=270$

\begin{tabular}{|c|l|}
\hline Educational Score & No. $(\%)$ \\
\hline Up to- 3 ( Below Primary level) & $1(0.37 \%)$ \\
\hline Up to- 4 (Primary Level) & $24(8.89 \%)$ \\
\hline Up to- 5 (Middle School Level) & $71(26.29 \%)$ \\
\hline Up to- 6 (high School Level) & $74(27.46 \%)$ \\
\hline$>$ 6 (College and above)) & $98(36.29 \%)$ \\
\hline
\end{tabular}

Table.7 Distribution of respondent according to their family type $n=270$

\begin{tabular}{|c|c|}
\hline Family Type & No.(\%) \\
\hline Joint & $125(46.34 \%)$ \\
\hline Nuclear & $143(53.66 \%)$ \\
\hline
\end{tabular}

Table.8 Distribution of respondents on the basis of Size of the Family n=270

\begin{tabular}{|c|c|}
\hline Size of Family & No.(\%) \\
\hline Up to- 3 & $34(12.59 \%)$ \\
\hline Up to- 4 & $56(20.74 \%)$ \\
\hline Up to- 5 & $43(15.92 \%)$ \\
\hline Up to- 6 & $35(12.96 \%)$ \\
\hline Up to- 7 & $11(4.07 \%)$ \\
\hline$>7$ & $91(33.70 \%)$ \\
\hline
\end{tabular}


Table.9 Distribution of respondents according to their Computer material possession $n=270$

\begin{tabular}{|c|c|c|c|c|c|c|c|c|c|c|c|}
\hline \multirow[t]{3}{*}{ Sl. No. } & \multirow[t]{3}{*}{ Material } & \multicolumn{10}{|c|}{ Distribution of Respondents } \\
\hline & & \multicolumn{2}{|l|}{ UG } & \multirow{2}{*}{$\begin{array}{c}\text { PG } \\
\text { Own }\end{array}$} & \multicolumn{3}{|c|}{ Ph. D } & \multicolumn{2}{|c|}{ Faculty } & \multicolumn{2}{|c|}{ Total } \\
\hline & & Own & Off & & Off & Own & Off & Own & Off & Own & Off \\
\hline 1. & Desktop & $\begin{array}{c}60 \\
(22.22 \%)\end{array}$ & $\mathbf{0}$ & $\begin{array}{c}22 \\
(8.14 \%)\end{array}$ & $\mathbf{0}$ & $4(1.48 \%)$ & $\mathbf{0}$ & $\begin{array}{c}24 \\
(8.89 \%)\end{array}$ & $47(17.40 \%)$ & $\begin{array}{c}110 \\
(40.74 \%)\end{array}$ & $47(17.40 \%)$ \\
\hline 2. & Laptop & $\begin{array}{c}40 \\
(14.81 \%)\end{array}$ & $\mathbf{0}$ & $\begin{array}{c}58 \\
(21.48 \%)\end{array}$ & $\mathbf{0}$ & $\begin{array}{c}30 \\
(11.11 \%)\end{array}$ & $\mathbf{0}$ & $\begin{array}{c}22 \\
(8.14 \%)\end{array}$ & $50(18.51 \%)$ & $\begin{array}{c}150 \\
(55.56 \%)\end{array}$ & $50(18.51 \%)$ \\
\hline 3. & Note Book & & $\mathbf{0}$ & & $\mathbf{0}$ & & $\mathbf{0}$ & & $2(\% 0.74)$ & & $2(\% 0.74)$ \\
\hline 4. & Modem & $\begin{array}{c}40 \\
(14.81 \%)\end{array}$ & $\mathbf{0}$ & $\begin{array}{c}52 \\
(19.25 \%)\end{array}$ & $\mathbf{0}$ & $\begin{array}{c}30 \\
(11.11 \%)\end{array}$ & $\mathbf{0}$ & $26(\%)$ & $47(17.40 \%)$ & $\begin{array}{c}148 \\
(54.81 \%)\end{array}$ & $47(17.40 \%)$ \\
\hline 5. & $\begin{array}{l}\text { Mobile } \\
\text { Phone }\end{array}$ & $\begin{array}{c}132 \\
(48.89 \%)\end{array}$ & $\mathbf{0}$ & $\begin{array}{c}61 \\
(22.59 \%)\end{array}$ & $\mathbf{0}$ & $\begin{array}{c}30 \\
(11.11 \%)\end{array}$ & $\mathbf{0}$ & $\begin{array}{c}47 \\
(17.40 \%)\end{array}$ & $\mathbf{0}$ & $270(100 \%)$ & $\mathbf{0}$ \\
\hline 6. & Smart Ph & $\begin{array}{c}75 \\
(\% 27.78)\end{array}$ & $\mathbf{0}$ & $54(20 \%)$ & $\mathbf{0}$ & $26(9.62 \%)$ & $\mathbf{0}$ & $\begin{array}{c}47(17.40 \\
\%)\end{array}$ & $\mathbf{0}$ & $\begin{array}{c}202 \\
(74.81 \%)\end{array}$ & $\mathbf{0}$ \\
\hline 7. & Total & $\begin{array}{c}347 \\
(128.51 \%)\end{array}$ & $\mathbf{0}$ & $\begin{array}{c}247 \\
(91.48 \%)\end{array}$ & 0 & $\begin{array}{c}120 \\
(44.44 \%)\end{array}$ & $\mathbf{0}$ & $\begin{array}{c}166(61.48 \\
\%)\end{array}$ & $\begin{array}{c}116 \\
(42.96 \%)\end{array}$ & $\begin{array}{c}880 \\
(325.92)\end{array}$ & $116(42.96 \%)$ \\
\hline
\end{tabular}

Note:- $\mathrm{OP}=$ Official Possession, $\mathrm{PP}=$ Personal Possession, $\mathrm{BH}=$ Both and $\mathrm{NO}=$ Never

Table.10 Distribution of respondent according to their family income $n=270$

\begin{tabular}{|c|c|c|}
\hline SL.NO. & Income Range & Distribution of Respondents \\
\hline 1. & Up to Rs. 250000 & $50(18.51 \%)$ \\
\hline 2. & Rs. $250000-$ Rs.500000 & $120(44.44 \%)$ \\
\hline 3. & Rs.500000- Rs.750000 & $37(13.70 \%)$ \\
\hline 4. & Rs.750000- Rs. 1000000 & $20(7.40 \%)$ \\
\hline 5. & Rs.1000000- Rs.1250000 & $19(7.03 \%)$ \\
\hline 6. & Above Rs.1250000 & $24(8.89 \%)$ \\
\hline
\end{tabular}

Table.11 Extent of Media Exposure of the Respondents n=270

\begin{tabular}{|l|c|c|c|c|c|}
\hline \multirow{2}{*}{$\begin{array}{c}\text { nedia } \\
\text { Exposure Gap Index }\end{array}$} & \multicolumn{5}{|c|}{ Distribution of Respondents } \\
\cline { 2 - 6 } & UG & PG & PhD & Faculty & Total \\
\hline $\mathbf{0 - 2 5}$ (Low) & $15(5.55 \%)$ & $29(10.73 \%)$ & $16(5.92 \% 0$ & $34(12.58 \%)$ & $94(34.78 \%)$ \\
\hline $\mathbf{2 6 - 5 0}$ (Medium) & $97(35.89 \%)$ & $26(9.62 \%)$ & $14(5.18 \%)$ & $13(4.81 \%)$ & $150(55.5 \%)$ \\
\hline $\mathbf{5 1 - 7 5}$ (Semi-Med) & $20(7.77 \%)$ & $6(2.22 \%)$ & 0 & 0 & $26(9.62 \%)$ \\
\hline $\mathbf{7 6 - 1 0 0}$ (High) & 0 & 0 & 0 & 0 & \\
\hline
\end{tabular}

Table.13 Average Time spend in computer per day by Respondents $n=270$

\begin{tabular}{|c|c|c|}
\hline \multicolumn{2}{|c|}{ Time spend in computer use on an average per day } & NO.(\%) \\
\hline $\mathbf{1 .}$ & More than two hours & $138(51.11 \%)$ \\
\hline $\mathbf{2}$ & One to two hours & $132(48.88 \%)$ \\
\hline $\mathbf{3}$ & Thirty minutes to one hour & 0 \\
\hline $\mathbf{4}$. & Less than thirty minutes & 0 \\
\hline $\mathbf{5}$ & Very occasional & 0 \\
\hline $\mathbf{6 .}$ & Never & 0 \\
\hline
\end{tabular}


Table.14 Distribution of Respondent by Type of Computer Work Done and Software Use n=270

\begin{tabular}{|c|c|c|c|}
\hline Sl. No & Types of work & Types of Software /Web used & No(\%) \\
\hline $\mathbf{1 .}$ & Word Processing & M.S. Word & $270(100 \%)$ \\
\hline $\mathbf{2 .}$ & Records Maintenance & M.S. Excel & $47(17.40 \%)$ \\
\hline $\mathbf{3 .}$ & Slides Preparation & M.S. PowerPoint & $124(45.92 \%)$ \\
\hline $\mathbf{4 .}$ & Web-designing & & \\
\hline $\mathbf{5}$ & Software developing & & \\
\hline $\mathbf{6 .}$ & Spread sheet & M.S. Excel & $60(22.22 \%)$ \\
\hline $\mathbf{7 .}$ & Analytical Work & M.S. Excel & $128(47.40 \%)$ \\
\hline $\mathbf{8 .}$ & $\begin{array}{c}\text { Preparation of } \\
\text { educational notes }\end{array}$ & M.S. Word & \\
\hline
\end{tabular}

Table.14A Extent of use of computer for different purposes $n=270$

\begin{tabular}{|c|c|c|c|c|c|}
\hline Index & UG & PG & PhD & Faculty & Total \\
\hline 0-25(Low) & 0 & $1(0.37 \%)$ & $3(1.11 \%)$ & $6(2.22 \%)$ & $11(4.07 \%)$ \\
\hline $\begin{array}{c}\text { 26-50 } \\
\text { (Medium) }\end{array}$ & $13(4.81 \%)$ & $30(11.1 \%)$ & $20(7.40 \%)$ & $33(12.21 \%)$ & $95(35.15 \%)$ \\
\hline $\begin{array}{c}\text { 51-75 (Semi- } \\
\text { Medium) }\end{array}$ & $92(34.04 \%)$ & $30(11.1 \%)$ & $7(2.59 \%)$ & $8(2.96 \%)$ & $137(50.69 \%)$ \\
\hline 76-100 (High) & $27(9.99 \%)$ & 0 & 0 & 0 & $27(9.99 \%)$ \\
\hline
\end{tabular}

Table.15 Distribution of Respondents on the basis of their Visit to Different Computer Service Provider Centre $n=270$

\begin{tabular}{|c|c|c|c|c|c|}
\hline Gap Index & UG & PG & PhD & Faculty & Total \\
\hline 0-25 (Low) & 0 & $1(0.37 \%)$ & 0 & 0 & $1(0.37 \%)$ \\
\hline $\mathbf{2 6 - 5 0}($ Medium) & $1(0.37 \%)$ & $9(3.33 \%)$ & 0 & 0 & $10(3.7 \%)$ \\
\hline $\begin{array}{c}\text { 51-75 (Semi- } \\
\text { Medium) }\end{array}$ & $13(4.81 \%)$ & $22(8.14 \%)$ & $13(4.81 \%)$ & $16(5.92 \%)$ & $63(23.31 \%)$ \\
\hline $\mathbf{7 6 - 1 0 0}($ High) & $118(43.66 \%)$ & $29(10.73 \%)$ & $17(6.29 \%)$ & $31(11.48 \%)$ & $195(72.15 \%)$ \\
\hline
\end{tabular}

Table.16 Distribution of Respondents on the Basis of their Nature and extent of using computer $\mathrm{n}=270$

\begin{tabular}{|c|c|c|c|c|c|}
\hline Gap Index & UG & PG & PhD & Faculty & Total \\
\hline 0-25 (Low) & 0 & $4(1.48 \%)$ & $5(1.85 \%)$ & $12(4.44 \%)$ & $21(7.78 \%)$ \\
\hline $\mathbf{2 6 - 5 0}$ (Medium) & $20(7.4 \%)$ & $34(12.58 \%)$ & $22(8.14 \%)$ & $24(8.88 \%)$ & $100(37 \%)$ \\
\hline $\begin{array}{c}\text { 51-75 (Semi- } \\
\text { Medium) }\end{array}$ & $104(38.48 \%)$ & $19(7.03 \%)$ & $3(1.11 \%)$ & $6(2.22 \%)$ & $131(48.47$ \\
\hline $\mathbf{7 6 - 1 0 0}($ High) & $8(2.96 \%)$ & $4(1.48 \%)$ & 0 & $6(2.22 \%)$ & $18(6.66 \%)$ \\
\hline
\end{tabular}


Table.17 Distribution of Respondents on the Basis of the Activities Undertaken by Use of Computer $\mathrm{n}=270$

\begin{tabular}{|c|c|c|c|c|c|}
\hline Gap Index & UG & PG & PhD & Faculty & Total \\
\hline 0-25 (Low) & 0 & $5(1.85 \%)$ & $3(1.11 \%)$ & $9(3.333 .7 \%)$ & $17(6.29 \%)$ \\
\hline $\mathbf{2 6 - 5 0}$ (Medium) & $18(6.66 \%)$ & $35(12.95 \%)$ & $22(8.14 \%)$ & $28(10.36 \%)$ & $103(38.14 \%)$ \\
\hline $\begin{array}{c}\text { 51-75 (Semi- } \\
\text { Medium) }\end{array}$ & $93(34.44 \%)$ & $21(7.77 \%)$ & $4(1.48 \%)$ & $10(3.7 \%)$ & $128(47.40 \%)$ \\
\hline $\mathbf{7 6 - 1 0 0}$ (High) & $21(7.77 \%)$ & $1(0.37 \%)$ & 0 & 0 & $22(8.14 \%)$ \\
\hline
\end{tabular}

Table 18 Distribution of Respondents on their Extent use of CAIs \& ICTs n=270

\begin{tabular}{|c|c|c|c|c|c|}
\hline Gap Index & UG & PG & PhD & Faculty & Total \\
\hline 0-25 (Low) & $2(0.74 \%)$ & $2(0.74 \%)$ & $4(1.48 \%)$ & $18(6.66 \%)$ & $26(9.62 \%)$ \\
\hline $\mathbf{2 6 - 5 0}($ Medium) & $2(0.74 \%)$ & $16(5.92 \%)$ & $13(4.81 \%)$ & $20(7.40 \%)$ & $51(18.87 \%)$ \\
\hline $\begin{array}{c}\text { 51-75 (Semi- } \\
\text { Medium) }\end{array}$ & $74(27.38 \%)$ & $34(12.58 \%)$ & $8(2.96 \%)$ & $6(2.22 \%)$ & $122(45.14 \%)$ \\
\hline 76-100 (High) & $54(19.98 \%)$ & $9(3.33 \%)$ & $3(1.11 \%)$ & $5(1.85 \%)$ & $71(26.27 \%)$ \\
\hline
\end{tabular}

Table.19 Purpose of use of ICT \& CAI services $n=270$

\begin{tabular}{|c|c|c|c|c|c|}
\hline Gap Index & UG & PG & PhD & Faculty & Total \\
\hline 0-25 (Low) & 0 & $1(0.37 \%)$ & $2(0.74 \%)$ & $3(1.11 \%)$ & $6(2.22 \%)$ \\
\hline $\mathbf{2 6 - 5 0}$ (Medium) & 0 & $3(1.11 \%)$ & $1(0.37 \%)$ & $6(2.22 \%)$ & $9(3.33 \%)$ \\
\hline $\begin{array}{c}\mathbf{5 1 - 7 5}(\text { Semi- } \\
\text { Medium) }\end{array}$ & $4(1.48 \%)$ & $27(9.99 \%)$ & $8(2.96 \%)$ & $13(4.81 \%)$ & $52(19.24 \%)$ \\
\hline $\mathbf{7 6 - 1 0 0}($ High) & $128(47.36 \%)$ & $30(11.1 \%)$ & $18(6.66 \%)$ & $26(9.62 \%)$ & $202(74.74 \%)$ \\
\hline
\end{tabular}

Table.20 Distribution of Respondents on the Basis of their Perception about Relative easiness of computer use $n=270$

\begin{tabular}{|c|c|c|c|c|c|}
\hline Gap Index & UG & PG & PhD & Faculty & Total \\
\hline 0-25 (Low) & $33(12.21 \%)$ & $13(4.81 \%)$ & $7(2.59 \%)$ & $3(1.11 \%)$ & $56(20.74 \%)$ \\
\hline $\mathbf{2 6 - 5 0}$ (Medium) & $75(27.75 \%)$ & $39(14.43 \%)$ & $20(7.4 \%)$ & $26(9.62 \%)$ & $160(59.25)$ \\
\hline $\mathbf{5 1 - 7 5}$ (Semi-Medium) & $12(4.44 \%)$ & $5(1.85 \%)$ & $2(0.74 \%)$ & $5(1.85 \%)$ & $24(8.88 \%)$ \\
\hline $\mathbf{7 6 - 1 0 0}$ (High) & $12(4.44 \%)$ & $5(1.85 \%)$ & 0 & $17(6.29 \%)$ & $34(12.58 \%)$ \\
\hline
\end{tabular}

Table.21 Distribution of Respondents on the Basis of their Perception about Advantage of Computer Use $n=270$

\begin{tabular}{|c|c|c|c|c|c|}
\hline Gap Index & UG & PG & PhD & Faculty & Total \\
\hline 0-25 (Low) & $14(5.18 \%)$ & $19(7.03 \%)$ & $11(4.07 \%)$ & $11(4.07 \%)$ & $55(20.37 \%)$ \\
\hline $\mathbf{2 6 - 5 0}$ (Medium) & $71(26.27 \%)$ & $38(14.16 \%)$ & $17(6.29 \%)$ & $19(7.03 \%)$ & $145(53.70 \%)$ \\
\hline $\begin{array}{c}\mathbf{5 1 - 7 5} \\
\text { (Semi-Medium) }\end{array}$ & $22(8.14 \%)$ & $3(1.11 \%)$ & $2(0.74 \%)$ & $7(2.59 \%)$ & $34(12.59 \%)$ \\
\hline $\mathbf{7 6 - 1 0 0}$ (High) & $25(9.25 \%)$ & $1(0.37 \%)$ & 0 & $10(3.70 \%)$ & $36(13.32 \%)$ \\
\hline
\end{tabular}


Table.22 Distribution of Respondents on their Perception about Usefulness of Computer in Job and Education $n=270$

\begin{tabular}{|c|c|c|c|c|c|}
\hline Gap Index & UG & PG & PhD & Faculty & Total \\
\hline 0-25 (Low) & $52(19.25 \%)$ & $28(10.36 \%)$ & $14(5.18 \%)$ & $26(9.62 \%)$ & $120(44.4 \%)$ \\
\hline $\mathbf{2 6 - 5 0}($ Medium) & $45(16.67 \%)$ & $22(8.14 \%)$ & $16(5.92 \%)$ & $15(5.56 \%)$ & $98(36.26 \%)$ \\
\hline $\begin{array}{c}\text { 51-75 (Semi- } \\
\text { Medium) }\end{array}$ & $3(1.11 \%)$ & $9(3.33 \%)$ & 0 & $2(0.74 \%)$ & $14(5.18 \%)$ \\
\hline $\mathbf{7 6 - 1 0 0}($ High) & $32(11.84 \%)$ & $2(0.74 \%)$ & 0 & $4(1.48 \%)$ & $38(14.06 \%)$ \\
\hline
\end{tabular}

Table.23 Distribution of Respondents on their Perception about Extent of Technological constraints $n=270$

\begin{tabular}{|c|c|c|c|c|c|}
\hline Gap Index & UG & PG & PhD & Faculty & Total \\
\hline $\mathbf{0 - 2 5}($ Low $)$ & $59(21.83 \%)$ & $26(9.62 \%)$ & $14(5.18 \%)$ & $13(4.81 \%)$ & $112(41.48 \%)$ \\
\hline $\mathbf{2 6 - 5 0}($ Medium) & $65(24.05 \%)$ & $16(5.92 \%)$ & $9(3.33 \%)$ & $14(5.18 \%)$ & $104(38.51 \%)$ \\
\hline $\begin{array}{c}\text { 51-75 (Semi- } \\
\text { Medium) }\end{array}$ & $4(1.48 \%)$ & $10(3.70 \%)$ & $4(\% 1.48)$ & $8(2.96 \%)$ & $26(9.62 \%)$ \\
\hline $\mathbf{7 6 - 1 0 0}($ High) & $4(1.48 \%)$ & $9(3.33 \%)$ & $3(1.11 \%)$ & $12(4.44 \%)$ & $28(10.37 \%)$ \\
\hline
\end{tabular}

Table.24 Distribution of Respondents on their Perception about Extent of Socio-psychological constraints $n=270$

\begin{tabular}{|c|c|c|c|c|c|}
\hline Gap Index & UG & PG & PhD & Faculty & Total \\
\hline $\mathbf{0 - 2 5}($ Low) & $25(9.25 \%)$ & $17(6.29 \%)$ & $3(1.11 \%)$ & $4(1.48 \%)$ & $49(18.13 \%)$ \\
\hline 26-50 (Medium) & $55(20.35 \%)$ & $18(6.66 \%)$ & $15(5.55 \%)$ & $10(3.7 \%)$ & $98(36.26 \%)$ \\
\hline $\begin{array}{c}\text { 51-75 (Semi- } \\
\text { Medium) }\end{array}$ & $32(11.84 \%)$ & $14(5.18 \%)$ & $8(2.96 \%)$ & $12(4.44 \%)$ & $66(24.42 \%)$ \\
\hline 76-100 (High) & $20(7.4 \%)$ & $12(4.44 \%)$ & $4(1.48 \%)$ & $21(7.77 \%)$ & $57(21.09 \%)$ \\
\hline
\end{tabular}

Table.25 Distribution of Respondents on their Perception about Extent of Institutional constraints $n=270$

\begin{tabular}{|c|c|c|c|c|c|}
\hline Gap Index & UG & PG & PhD & Faculty & Total \\
\hline 0-25 (Low) & $43(15.91 \%)$ & $18(6.66 \%)$ & $12(4.44 \%)$ & $15(5.55 \%)$ & $88(32.56 \%)$ \\
\hline $\mathbf{2 6 - 5 0}($ Medium) & $60(22.2 \%)$ & $27(9.99 \%)$ & $10(3.7 \%)$ & $16(5.92 \%)$ & $113(41.81 \%)$ \\
\hline $\begin{array}{c}\text { 51-75 (Semi- } \\
\text { Medium) }\end{array}$ & $22(8.14 \%)$ & $10(3.7 \%)$ & $3(1.11 \%)$ & $10(3.7 \%)$ & $45(16.25 \%)$ \\
\hline 76-100 (High) & $7(2.59 \%)$ & $6(2.22 \%)$ & $4(1.48 \%)$ & $7(2.59 \%)$ & $24((8.88 \%)$ \\
\hline
\end{tabular}


Table.26 Extent of Computer Use by the Respondents and their Efficiency n=270

\begin{tabular}{|c|c|c|c|}
\hline Sl. No. & Aspects & $1^{\text {st }}$ Majority & $2^{\text {nd }}$. Majority \\
\hline 1 & First contact with computer & During UG $(40.74 \%)$ & Below HS (29.62\%) \\
\hline 2. & Reason for computer use & $\begin{array}{l}\text { Course at School } \\
\quad(29.62 \%)\end{array}$ & $\begin{array}{l}\text { Available at school } \\
\qquad(29.62 \%)\end{array}$ \\
\hline 3 & Purpose of Using Computer & $\begin{array}{l}\text { For getting experience } \\
\qquad(82.59 \%)\end{array}$ & $\begin{array}{l}\text { For professional } \\
\text { excellence }(17.4 \%)\end{array}$ \\
\hline 4 & Daily time spend on Computer & $\begin{array}{l}\text { More than two hours } \\
(51.11 \%)\end{array}$ & $\begin{array}{l}\text { One to two hours } \\
\quad(48.88 \%)\end{array}$ \\
\hline 5 & Software used & MS Word (100\%) & $\begin{array}{l}\text { MS Power Point } \\
\quad(45.92 \%)\end{array}$ \\
\hline 6 & $\begin{array}{l}\text { Extent of Computer use for different } \\
\text { purposes }\end{array}$ & Medium level (50.69\%) & $\begin{array}{l}\text { Semi-medium level } \\
(35.15 \%)\end{array}$ \\
\hline 7 & $\begin{array}{l}\text { Extent of visit to computer service } \\
\text { provider }\end{array}$ & Low $(72.15 \%)$ & Medium $(23.31 \%)$ \\
\hline 8 & Nature and extent of computer use & Medium $(48.47 \%)$ & Semi-medium (37\%) \\
\hline 9 & $\begin{array}{l}\text { Extent of computer use for different } \\
\text { purposes }\end{array}$ & Medium (47.4\%) & Semi-medium $(38.14 \%)$ \\
\hline 10 & Extent of use of ICT /CAI & Semi-medium $(45.14 \%)$ & $\operatorname{Low}(26.17 \%)$ \\
\hline 11 & Purpose of use ICT /CAI & Low $(74.74 \%)$ & Medium $(19.24 \%)$ \\
\hline 12 & Perception of relative easiness & Semi-medium $(59.25 \%)$ & High $(20.74 \%)$ \\
\hline 13 & Perception of extent of advantages & Semi-medium $(53.7 \%)$ & High $(20.37 \%)$ \\
\hline 14 & Perception of usefulness & High $(44.4 \%)$ & Semi-medium $(36.26 \%)$ \\
\hline 15 & Perception of technological constraints & High $(41.48 \%)$ & Semi-medium $(38.51 \%)$ \\
\hline 16 & $\begin{array}{l}\text { Perception of socio-psychological } \\
\text { constraints }\end{array}$ & Semi-medium $(36.26 \%)$ & Medium $(24.42 \%)$ \\
\hline 17 & Perception of institutional constraints & Semi-medium $(41.81 \%)$ & High $(32.56 \%)$ \\
\hline
\end{tabular}

From the above Table 11 can be observed that a majority of the UG respondents $(35.89 \%)$ fall in the medium level of gap index in media exposure. In case of PG students it was found almost a same percentage of respondents fall in the Low and Medium level of media exposure gap $(11.11 \%$ and $9.26 \%$ respectively). In case of $\mathrm{Ph}$. D and faculty group it was found that all the respondents fall in the low and medium level of media exposure gap index. When taking the total respondents into account it was found that $55.5 \%$ (150) respondents fall in the medium level of gap index followed by $34.78 \%$ in low gap index category, and $9.62 \%$ fall in the semi-medium category of gap index. As lower is the gap means higher is the exposure, it can be summarized that majority of the respondents had semi-medium to high level of media exposure.

\section{Daily time spend on computer}

When considering the daily time spend on computer for different purposes it was found from the table 13 that $51.11 \%$ were using computer for more than two hours daily followed by $48.88 \%$ uses computer for one to two hours daily.

Further efforts were taken for ascertaining the extent of use of computer for different purposes. For that word processing, record maintenance, slide presentation, web designing, software development, spread sheet operation, analytical work, preparation of educational notes have been considered as different purposes and respondents were asked to mention their extent of use of computer for all those purposes on a four point scale. Gap Index was calculated for 
each respondent and on the basis of the index value respondents were distributed in four class intervals as presented in table 14A.

From the above table 14A it can be observed that a majority of respondents 92 (34.04\%), 27 respondents $(9.99 \%)$ and 13 respondents (4.81\%) under UG followed by PG majority of 300 respondents $(11.1 \%)$ in both of the index range 26-50 and 51-75, respondents 1 $(0.37 \%)$ were having Information on extent of use of computer for different purposes. It is also observed that a majority of 19 respondents $(7.03 \%), 7$ respondents $(2.59 \%)$ and 4 respondents (1.48) under $\mathrm{PhD}$ followed by Faculty member majority of 33 respondents (12.21\%), and 6 respondents $(2.22 \%)$ were having Information on extent of use of computer for different purposes. As low gap indicate high level of use and vice versa, it can be said that respondents were using computer for different purposes to the extent of medium to semi-medium level.

From the above table it can be observed that a $72.15 \%$ of the total respondents were having high gap in this respect followed by $23.31 \%$ having semi-medium gap which means respondents were having low to medium extent visit to different service provider centre.

\section{Nature and extent of using computer}

To ascertain the nature of extent of using computer respondents were asked to mention the different purposes of using computer and the frequency thereof. Eight different types of purposes have been considered and the frequency was measured in a 4 point scale. Gap Index was calculated by using the formula as presented in the methodology chapter. Table 16 represents the percentile distribution of respondents in four class intervals.
From the above table it can be observed that a majority of the total respondents $(48.47 \%)$ fall in the semi-medium gap category followed by medium level gap category $(37 \%)$. Only $7.78 \%$ was found in low gap category whereas $18 \%$ was found in high gap category. Results amply establishes that respondents were using computer to the extent of semimedium to high level.

From the above table it can be observed that a majority of the total respondents $(47.4 \%)$ had semi-medium level gap followed by $38.14 \%$ having medium level of gap. Only $8.14 \%$ was found to have high gap in this regard and $6.29 \%$ was found to have low gap which means respondents undertake computer based activities to the tune of semi-medium to medium level.

From the above table it can be observed that a majority of the total respondents (45.14\%) had semi-medium level of gap followed by $26.27 \%$ having high level of gap, $18.87 \%$ having medium level gap and $9.62 \%$ was found to have low gap in using different ICT and CAI portals respectively.

From the above table it can be observed that out of the total respondents, majority of respondents $(74.74 \%)$ had high gap in using different ICT/CAI portals followed by semimedium, medium and low gap index which means the rate of use of ICT by the respondents were low.

From the above Table it can be observed that of the total respondents $53.7 \%$ perceived medium advantage gap followed by $20.37 \%$ perceived low gap in advantages of using computer. As a whole it can be said that all the respondents perceived high advantages of computer use.

From the above Table it can be observed that a majority of the total respondents (44.4\%) perceived low gap in easiness in computer use 
followed by $36.26 \%$ found to perceived medium level gap in easiness which means respondents perceived high level of usefulness of computer.

From the above table it can be observed that a majority of the total respondents $(41.48 \%)$ perceived low gap in technological constraints followed by $38.51 \%$ perceived medium level technological constraints. Further $9.26 \%$ and $10.73 \%$ of the total respondents perceived semi-medium and high level of technological constraints gap respectively. That means respondents perceived high to semi-medium level of technological constraints in computer use.

\section{Extent of Socio-psychological constraints}

From the above table 23 it can be observed that a majority of the total respondents $(36.26 \%)$ perceived medium level sociopsychological constraints gap followed by $24.42 \%, 21.09 \%$ and $18.13 \%$ perceived semimedium, high and low level of sociopsychological constraints gap respectively.

\section{Extent of institutional constraints}

From the above table 24 it can be observed that a majority of the total respondents $(41.81 \%)$ perceived medium level of institutional constraints gap followed by $32.56 \%, 16.25 \%$ and $8.88 \%$ perceived low, semi-medium and high level of institutional constraints gap respectively. That means respondents perceived semi-medium to high level of institutional constraints.

\section{Summary of Extent of Computer Use by the Respondents and their Efficiency}

The summary of the results with regard to extent of computer use by the respondents and their efficiency is presented below in tabular form.
In conclusion the present study implied that although computer has become integral part of education and professional activities but there are much scope for improvement of the status of computer use by the students and academicians of agricultural science. Nowadays traditional agriculture in general and agricultural extension in particular is transforming from its traditional mode to digital mode. Our country is also trying to transform into digital mode. For that matter human resources, like students - who are the future professional must be capable enough to operate through digital mode. Academicians are also to be efficient enough to teach the student properly in this regard. Present research work was limited to one institute which can be conducted among the students and academicians of many other institutions to have a clear picture of different regions as well as the entire country. Even more variables could be considered for more detailed study. The results of such detailed study would have helped in designing the course curriculum as well as for in service proficiency training.

\section{References}

Al-Shayaa, M. S. 2011. Constraints to use computers among agricultural extension workers in Riyadh and Qaseem regions of Saudi Arabia, The Journal of Animal \& Plant Sciences, ISSN: 1018-7081, 21(2), pp: 264-268.

Gagandeep Kaur Grewal, A.S. Gill and K.S. Jyoti (2015), Review Paper on Comprehensive Role of Computer Applications in Agriculture, International Journal of Advanced Rsearch in Computer Science and Software Engineering, 5 (3): 345-347.

Sujai Das; D. Nag and Laxmikanta Nayak. (2011). Development of ICT in Agriculture in India, NIRJAFT, 12 Regent Park, Kolkata. 
Vekiri Ioanna. 2009. Socioeconomic differences in elementary students' ICT beliefs and out-of-school experiences, www.elsevier.com/locate/compedu.pp: 947.

Zamfir Andreea.(2008). Impact of Using Computer Applications in Education on
Teaching-Learning Process, 7th WSEAS Int. Conf. on Applied Computer \& Applied Computational Science (ACACOS '08), Hangzhou, China ,ISBN: 978-960-6766-49-7.pp: 688.

\section{How to cite this article:}

Suman Saha, S. D. Mukhopadhyay, K. Pradhan and Acharya, S. K. 2021. Exploring the Perception of Information Communication Tools Application in Agricultural Education. Int.J.Curr.Microbiol.App.Sci. 10(01): 2896-2909.

doi: https://doi.org/10.20546/ijcmas.2021.1001.336 